\title{
The Use Of Tocilizumab In Combination With Methotrexate In Indonesian Rheumatoid Arthritis Patients (PICTURE INA Study)
}

\author{
Bambang Setyohadi ${ }^{1}$, Harry Isbagio ${ }^{1}$, Rachmat Gunadi Wachjudi ${ }^{2}$, Joewono Soeroso ${ }^{3}$, \\ Handono Kalim ${ }^{4}$, Dedy Nur Wachid Achadiono 5
}

${ }^{1}$ Cipto Mangunkusomo Hospital, Jakarta,

${ }^{2}$ Hasan Sadikin Hospital, Bandung,

${ }^{3}$ Soetomo Hospital, Surabaya,

${ }^{4}$ Dr. Saiful Anwar Hospital, Malang,

${ }^{5}$ Sardjito Hospital, Yogyakarta

\author{
A R T I C L E I N F O \\ Keywords: \\ Rheumatoid Arthritis \\ Tocilizumab \\ DMARD \\ DAS28

\begin{abstract}
Corresponding author:
Bambang Setyohadi MD

E-mail address:

b_setiyohadi@yahoo.com

All authors have reviewed and approved the final version of the manuscript.
\end{abstract}

https://doi.org/10.37275/IJR.v10i1.1

\section{Introduction}

Rheumatoid arthritis (RA) is a progressive, systemic autoimmune disease characterized by inflammation of the synovial membrane lining the joints. This

\begin{abstract}
A B S T R A C T
Background Aim of this research is to assess the efficacy and safety of tocilizumab (TCZ) in combination with methotrexate (MTX) in Indonesian patients with moderate to severe active rheumatoid arthritis (RA) who have an inadequate response to nonbiologic DMARDs. Methods This was a interventional, prospective, single arm, multicenter, study in Indonesian male or female patients aged $\geq 18$ years old, with a diagnosis of RA for $>6$ months based on ACR 1987 revised criteria with moderate to severe disease activity (DAS28 score $>3.2$ ) after $\geq 12$ weeks of non-biologic DMARDs treatment. The treatment consisted of tocilizumab, $8 \mathrm{mg} / \mathrm{kg}$, intravenous (IV), every 4 weeks for a total of 6 infusion in combination with oral MTX (10-25 mg) every week. Efficacy was assessed based on the percentage of patients achieving low disease activity state (DAS28 < 3.2), percentage of patients achieving reduction $\geq 1.2$ point of DAS28, percentage of patients achieving remission (DAS28 $<2.6$ ), and percentage of patients with ACR20, ACR50, and ACR70 responses. Descriptive statistics will be used for presentation of results. Results $100 \%$ patients reached low disease activity (DAS28 $\leq 3.2$ ) at last study visit (week 24) and clinically significant improvement (reduction at least 1.2 units) at every visit in DAS28, both for ITT or PP patients. Remission (DAS28 < 2.6) was observed in $82.1 \%$ (ITT patients) and $93.1 \%$ (PP patients) on last study visit. ACR20, ACR50, and ACR70 were achieved in 20\%, 34\%, and 34\% (ITT patients), and $7 \%, 24 \%$, and $62 \%$ (PP patients) on week 24. There were 3 out of 39 patients $(7.69 \%)$ with adverse events (AE) and serious adverse events (SAE) that resulted in discontinuation of TCZ treatment, consisting of 1 patient with SAE of sepsis ec acquired community pneumonia, 1 patient with SAE of pneumonia tuberculosis, and 1 patient with $\mathrm{AE}$ of candidiasis. Most common adverse events were hepatic dysfunction $(30.7 \%)$, hypercholesterolemia $(23.1 \%)$, followed by arthralgia $(20.5 \%)$ Twelve percent of patients needed dose modification due to elevated liver enzyme (elevated ALT/SGPT level). Conclusion Tocilizumab seems to be efficacious and likely to have good safety profile in non- biologic DMARD nonresponsive RA patients of PICTURE INA study.
\end{abstract}


joint destruction and disability. RA is the most common chronic inflammatory disease in the western world with a prevalence of $0.5 \%$ to $1.0 \%{ }^{1}$.

Current treatment in RA such as NSAID, DMARD and biological compounds that target TNF-a, B-cells or T-cells had some limitation of effectiveness in many patients $2,3,4,5$, hence there is a medical need for more effective treatments for RA based on an understanding of the underlying pathophysiology of the course of the disease.

IL-6 is a multifunctional pro inflammatory cytokine produced by a variety of cell types. IL- 6 has been implicated in the pathogenesis of a variety of diseases including inflammatory rheumatic diseases, osteoporosis, neoplasia, and aging.6,7 IL-6 exerts its effects through a ligand-specific receptor (IL-6R), present both in soluble and membrane-expressed forms. Elevated serum and synovial IL-6 levels have been reported in RA patients compared with controls reflecting local production of IL- 6 by the synovium. ${ }^{6}$ Preclinical studies suggest that IL- 6 is associated with demargination of neutrophils and egress from the bone marrow so that the opposite effect may occur with IL-6R signaling inhibition. IL-6 levels correlate with disease activity in $R A, 8$ and improvements associated with DMARD use are accompanied by a reduction in serum IL-6 levels. ${ }^{7}$

Tocilizumab (TCZ) is a recombinant humanized anti-human monoclonal antibody of the immunoglobulin IgG $_{1}$ subclass directed against the IL6 receptors and produced by recombinant DNA technology. Administration of TCZ is expected to inhibit all IL- 6 related negative effects in RA patients. The present study is intended to assess the efficacy, safety and tolerability of TCZ in combination with methotrexate (MTX) in patients with moderate to severe active $\mathrm{RA}$ in a routine clinical practice setting in Indonesian patients.

\section{Research Methods}

This study was using an interventional, single-arm, multicenter clinical study conducted from March 2011 to July 2012 in Indonesian patients, aged $\geq 18$ years, suffering from rheumatoid arthritis for more than 6 months duration based on ACR 1987 revised criteria. The patients have moderate to severe disease activity (DAS28 > 3.2) at screening and still have active RA after $\geq 12$ weeks of non-biologic DMARDs (methotrexate, chloroquine, hydroxychloroquine, parenteral gold, sulfasalazine, azathioprine, and leflunomide). DMARD dose should have been stable for a period of 8 weeks or longer; but have inadequate clinical response.

Excluded from the study were patients with the following criteria: major surgery (including joint surgery) within 8 weeks prior to screening or planned to have major surgery within 6 months following enrollment; autoimmune disease other than RA including systemic lupus erythematosus (SLE), mixed connective tissue disease (MCTD), scleroderma, polymyositis, or significant systemic involvement secondary to RA (e.g. vasculitis, pulmonary fibrosis or Felty's syndrome); prior history of or current inflammatory joint disease other than RA (e.g. gout, reactive arthritis, psoriatic arthritis, seronegative spondyloarthropathy, lyme disease). Those receiving the following drugs were also excluded: any investigational agent or calcineurin inhibitors (e.g. tacrolimus or cyclosporine), mycophenolate mofetil or mycophenolic acid sodium within 4 weeks (or 5 halflives of drug, whichever is longer); previous treatment with any biologic drug that is used in the treatment of $\mathrm{RA}$, treatment with IV gamma globulin, plasmapheresis within 6 months before the study; intra articular or parenteral corticosteroids within previous 6 weeks; immunization with a live/attenuated vaccine within 4 weeks prior to baseline; previous treatment with TCZ; any previous treatment with alkylating agents, such as cyclophosphamide or chlorambucil, or with total lymphoid irradiation. Renal dysfunction (serum creatinine $>1.6 \mathrm{mg} / \mathrm{dL}$ for female or $1.9 \mathrm{mg} / \mathrm{dL}$ for male), liver dysfunction (serum transaminases $>1.5$ ULN), anemia with hemoglobin $<8 \mathrm{~g} / \mathrm{dL}$, leucopenia (WBC $<300 / \mathrm{mm}^{3}$ ), thrombocytopenia (platelet count < $100.000 / \mathrm{mm}^{3}$ ), and positive $\mathrm{HBsAg}$, anti $\mathrm{HCV}, \mathrm{HIV}$, 
tuberculosis within 3 years prior to the study, were also excluded.

The protocol for this study was reviewed and approved by Ethical Committee of Faculty of Medicine of University of Indonesia, Jakarta. All participating subjects in this study signed the informed consent for participation in the study.

\section{Study treatment}

Patients receive an intravenous infusion of $8 \mathrm{mg} / \mathrm{kg}$ of tocilizumab every 4 weeks on an outpatient basis for a total of 6 infusions (week 0, week 4, week 8, week 12, week 16 and week 20). For patients weighing more than $100 \mathrm{~kg}$, the maximum dose of TCZ will be $800 \mathrm{mg}$. Oral methothrexate (MTX) is continued with the range dose of 10-25 mg every week, according to previous stable dose of the patient. The dose and route of MTX is to be continued without change unless an adjustment is necessary for safety reasons. Schematic representation of treatment and follow up can be seen in figure 1 .

Forty patients were planned to be enrolled in this study. There was no sample size calculation due to exploratory nature of the study. The number of subjects enrolled in the study was determined based on practical considerations in study site according to their potential for enrollment.

\section{Efficacy evaluation}

Efficacy of treatment was measured in intent to treat (ITT) patients, i.e all patients, who take at least one dose of study drug and return at least once after receiving one dose. Per protocol (PP) patients who completed TCZ treatment of six doses, without eligibility violation will also be evaluated.

The following parameters will be evaluated are: 1) number and percentage of patients achieving low disease state (DAS28 < 3.2), 2) number and percentage of patients achieving significant improvement (reduction of at least 1.2 units of DAS28) and time to clinically significant improvement in DAS28, 3) number and percentage of patients achieving remission (DAS28 <2.6) at every visit, 4) disease activity as measured by
American College of Rheumatology 20\%, 50\%, and 70\% improvement (ACR 20/50/70) were measured at week $24,5)$ Laboratory improvement in term of CRP and ESR was also evaluated.

The DAS28 is a combined index for measuring disease activity in RA. The index includes swollen and tender joint counts, acute phase response (ESR or CRP), and general health status. The DAS28, which uses a 28 joint count, is derived from the original DAS, which includes a 44 swollen joint count. The index is calculated using the following formula: DAS28 $=0.56 \times$ $\sqrt{ }(\mathrm{TJC})+0.28 \times \sqrt{ }(\mathrm{SJC})+0.70 \times \ln (\mathrm{ESR})+0.014 \times \mathrm{GH}$ which TJC $=$ tender joint count on 28 joints, SJC $=$ swollen joint count on 28 joints, ln = natural log, ESR $=$ erythrocyte sedimentation rate $(\mathrm{mm} / \mathrm{hr})$, and $\mathrm{GH}=$ general health, i.e. patient's global assessment of disease activity (100 mmVAS). The DAS2 8 scale ranges from 0 to 10 which higher scores represent higher disease activity.

\section{Safety evaluation}

All patients who receive at least one dose of the study drug and return at least once after receiving study drug will be subject to safety evaluation. Parameters of safety will include incidence of adverse events, serious adverse events, and adverse event of special interest (e.g. ALT (SGPT) or AST (SGOT) elevations, serum lipids elevations).

\section{Data analysis}

This is an interventional, single arm, multicenter study. Primary endpoint and other end points are reported as descriptive statistics. Thirty nine patients were enrolled from March 20, 2011 July 4, 2012.

\section{Results}

\section{Disposition of patients}

Fifty eight patients were screened and 39 patients were enrolled to this study and included for evaluation of safety. Four patients were withdrawn during the study due to SAE (3 patients) and treatment failure (1 
patient). There were 7 patients who had protocol violations related with the entry criteria (eligibility criteria), in which 6 patients completed the study until week 24 due to delay in identifying the protocol deviation related to the eligibility criteria while the other patient was one of the four patients withdrawn during the study. Thus, only 29 patients included as per protocol (PP) evaluation. Patient disposition is presented in figure 2.

\section{Efficacy Evaluation}

1. Proportions of patients achieving low disease activity (DAS28 $\leq 3.2$ )

The efficacy of tocilizumab (TCZ) in combination with MTX was observed based on achievement of low disease activity DAS28 (DAS28 $\leq 3.2$ ). The patients in this study showed good efficacy result as measured by DAS28 that decreased over time. For ITT populations at week 24, mean of DAS28 value was 1.75, (ranging 0.3 to 2.9), while for PP patients, mean of DAS28 value was 1.8 (ranging 0.49 to 2.9 ).

In some patients, this low disease state has been attained since week 4 (after one dose of tocilizumab injection).

2. Achievement of clinically significant improvement (Reduction of DAS28 at least 1.2 unit):

In ITT patients, a clinically significant improvement (reduction at least 1.2 units at every visit) in DAS28 was observed in $87.2 \%$ patient at week-4, 91.2\% at week-8, 94.7\% at week-12, 97.3\% at week-16 and week-20. At week-24, all patients (100\%) have significant improvement. A clinically significant improvement was observed in the PP population, $93.1 \%$ of patients at week-4, 96.4\% at week-8, and all patients achieved $100 \%$ improvement at week12, 16, 20 and 24 (Figure 3). Overall, large majority of patients only required a single infusion in order to achieve a clinically significant improvement (reduction at least 1.2 units) in DAS28.

3. Number and percentage of patients achieving remission (DAS28 < 2.6) at every visit and time to DAS28 remission.

In ITT population there were $20.5 \%$ of patients achieving remission (DAS28<2.6) by week four, which increased at subsequent visits as shown in Figure 4. $36.8 \%$ patients were achieving remission by week $8,44.7 \%$ by week $12,59.0 \%$ by week 16 , $74.4 \%$ and $82.1 \%$ by week 20 and 24 . However in PP patients, there were $13.8 \%$ patients achieved remission by week $4 ; 34.5 \%$ by week $8 ; 37.9 \%$ by week $12 ; 55.2 \%$ by week $16 ; 86.2 \%$ by week 20 and $93.1 \%$ by week 24 .

4. Percentage of patients with ACR20, ACR50, and ACR70 response at week 24

For ACR response on ITT patient population, ACR20 has been achieved in $20 \%$ patients, while on ACR50 and ACR70 have been achieved in 34\% patients on week 24. PP population showed that ACR20, ACR50, and ACR70 have been achieved in 7\%, 24\% and $62 \%$ patients, respectively. However, there are $12 \%$ patients as per ITT and 7\% as per PP did not response by week 24 (figure 5). The classification of patient's response was made based on nonoverlapping ACR response rate categories, instead of cumulative categories. The patients with ACR50 were not included in calculation of percentage of patients with ACR20 and patients with ACR 70 were not included in calculation of patients with ACR50 and ACR20.

5. ESR and CRP at every visit

ESR and CRP are important markers used for diagnosis and monitoring of disease activity and response to anti-inflammatory therapy. Mean of hsCRP and ESR were normalized (0-20 mm \& hs CRP $\leq 10 \mathrm{mg} / \mathrm{L}$ ) after first infusion and stable until last follow up at week-24 both for ITT and PP patient population (Figure 6)

\section{Safety Evaluation}

Common adverse events can be seen in table 3 . The most frequent adverse events are increased hepatic 
enzyme $(30.7 \%)$, followed by hypercholesterolemia $(23.1 \%)$ and arthralgia (20.5\%).

There were 3 out of 39 patients (7.69\%) with adverse events (AE) and serious adverse events (SAE) that resulted in discontinuation of TCZ treatment, consisting of 1 patient with SAE of sepsis ec acquired community pneumonia, 1 patient with SAE of pneumonia tuberculosis, and 1 patient with $\mathrm{AE}$ of candidiasis.
Most common adverse event special interest (AESI) was hepatic events (13 events), followed by infection which was treated with anti-infection (2 events) and bleeding event (1 event). There were 2 patients indicated as withdrawn patients however all cases have been resolved at the time of study closed.

Figure 7 shows lipid profiles of patients treated with TCZ and MTX in every visit. There were increases of total and LDL cholesterol, while triglyceride and HDL were relatively unchanged.

Figure 1. Schematic representation of treatment with TCZ and MTX.

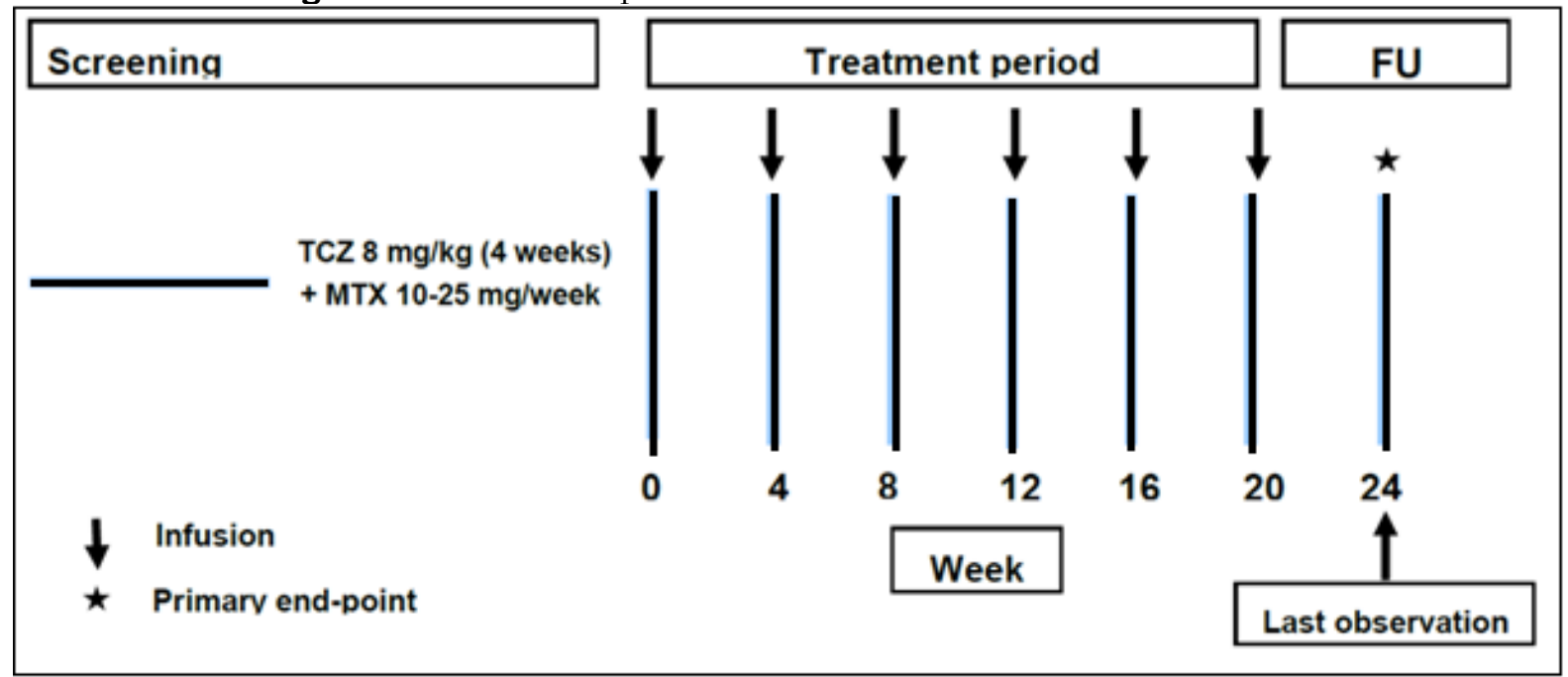


Figure 2. Patient disposition of study subjects

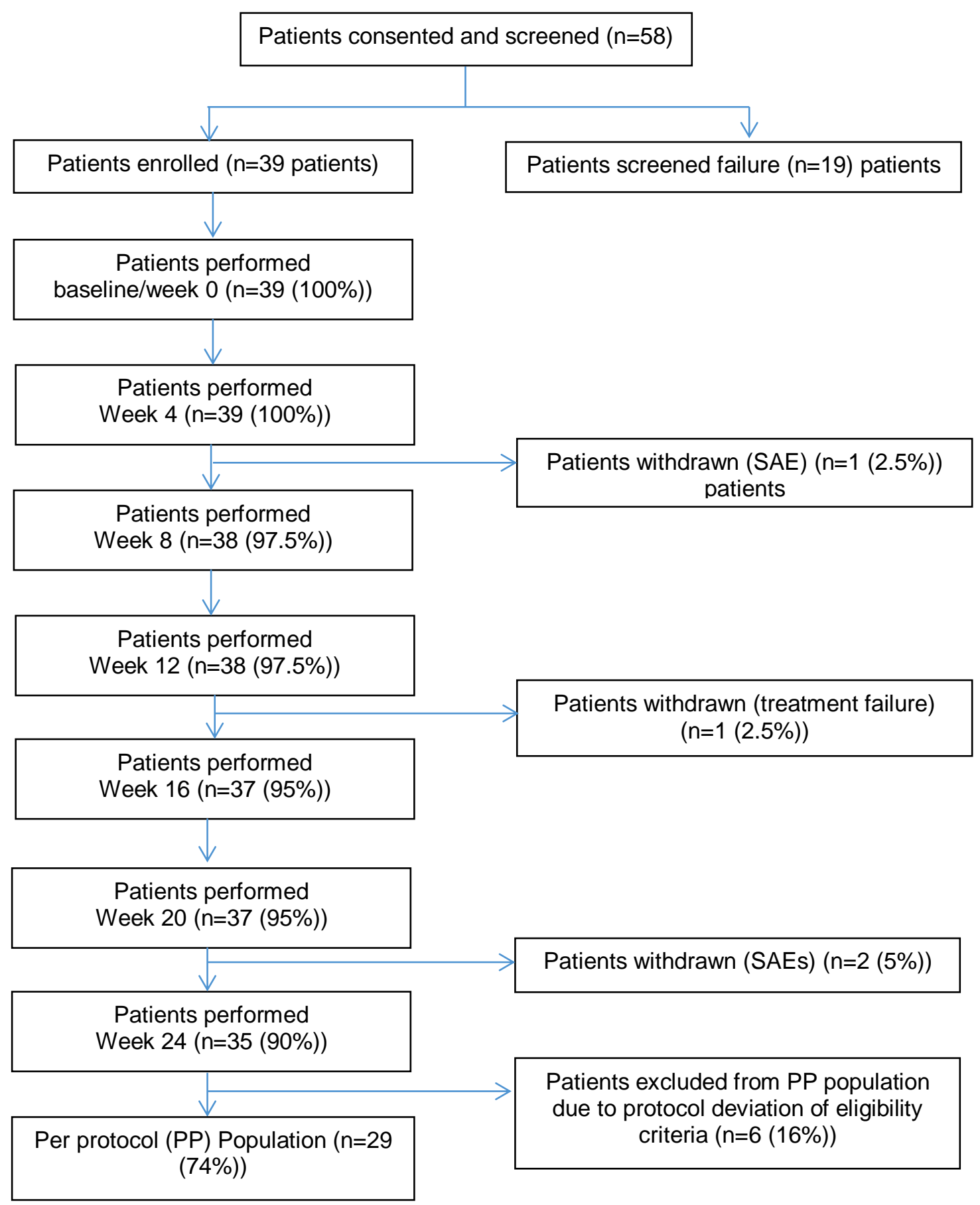

Characteristics data of the study subjects can be seen in table 1 . Most of patients were female, and more than half were less than 50 years old. Ethnicity was dominated by those from Java island (Javanese and Sundanese). 
Table 1. Demographic Characteristic and baseline data of study subjects

\begin{tabular}{|c|c|c|c|}
\hline Variable & n (\%) & Mean \pm SD & Median (min-max) \\
\hline \multicolumn{4}{|l|}{ Gender } \\
\hline Male & $3(7.7)$ & & \\
\hline Female & $36(92.3)$ & & \\
\hline Age & & & $53(19-75)$ \\
\hline$>50$ yrs & 17 (43.6) & & \\
\hline$<50$ yrs & $22(56.4)$ & & \\
\hline \multicolumn{4}{|l|}{ Ethnic } \\
\hline Javanese & $23(59.0)$ & & \\
\hline Sundanese & $8(20.5)$ & & \\
\hline Chinese & $3(7.7)$ & & \\
\hline Bataknese & $2(5.1)$ & & \\
\hline Padangnese & $2(5.1)$ & & \\
\hline Javanese-Sundanese & $1(2.6)$ & & \\
\hline Length of disease & & & $3(1-31)$ \\
\hline$\geq 5 \mathrm{yrs}$ & $11(28.2)$ & & \\
\hline$<5$ yrs & $28(71.8)$ & & \\
\hline $\mathrm{SBP}(\mathrm{mmHg})$ & & $119.6+11.2$ & \\
\hline DBP (mmHg) & & $78.6 \pm 6.5$ & \\
\hline \multicolumn{4}{|l|}{$\begin{array}{l}\text { Previous } R A \text { related } \\
\text { treatments }\end{array}$} \\
\hline Methotrexate & $36.6 \%$ & & \\
\hline Folic acid & $30.8 \%$ & & \\
\hline Chloroquine & $4.8 \%$ & & \\
\hline Calcium & $1.9 \%$ & & \\
\hline Celecoxib & $1.9 \%$ & & \\
\hline Lab parameters & & Mean + SD & Min - Max \\
\hline ESR (mm) & & $48.1 \pm 26.10$ & $9-105$ \\
\hline CRP (mg/L) & & $17.6 \pm 21.58$ & $0.25-77$ \\
\hline Hemogloblin (g/dL) & & $12.2 \pm 1.29$ & $8.3-14$ \\
\hline Platelet (x103) & & $351.3 \pm 116.6$ & $41.1-594$ \\
\hline ANC & & $6.26 \pm 2.4$ & $2.58-11.51$ \\
\hline Total Cholesterol & & $179.8 \pm 37.5$ & $94-275$ \\
\hline Triglyceride & & $102.3+43.9$ & $45-211$ \\
\hline HDL Chol. (mg/dL) & & $56.8 \pm 11.5$ & $37-82$ \\
\hline LDL Chol. (mg/dL) & & $105.9+28.7$ & $47-178$ \\
\hline SGOT (mg/dL) & & $18.3 \pm 7.5$ & $7-46$ \\
\hline SGPT (mg/dL) & & $17.7 \pm 8.8$ & $4-50$ \\
\hline
\end{tabular}

Note: SBP: Systolic Blood Pressure; DBP: Diastolic Blood Pressure; ESR: Erithrocyte Sedimentation Rate, CRP: C-Reactive Protein, ANC: Absolute Neutrophyl Count, HDL: High Density Lipoprotein; LDL: Low Density Lipoprotein; SGOT: Serum Glutamic-Oxaloasetic Transaminase, SGPT: Serum Glutamic Pyruvic Transaminase.

Table 2. Percentage of patients achieving low disease activity (DAS28<3.2) on each time visit based on ITT and PP patients.

\begin{tabular}{lccc}
\hline & & \multicolumn{2}{c}{$\%$ of DAS28 $<\mathbf{3 . 2}$} \\
& $\mathbf{N}$ & ITT & PP \\
\hline Baseline & 39 & 0 & 0 \\
W-4 & 39 & 30.8 & 27.6 \\
W-8 & 38 & 48.7 & 48.3 \\
W-12 & 38 & 69.2 & 72.4 \\
W-16 & 37 & 82.1 & 86.2
\end{tabular}




\begin{tabular}{llll}
$\mathrm{W}-20$ & 37 & 89.7 & 96.6 \\
$\mathrm{~W}-24$ & 35 & 100 & 100 \\
\hline
\end{tabular}

Figure 3. Percentage of patients achieving clinically significant improvement (DAS28 reduction of more than 1.2) during each visit in ITT patients (open bar) and PP patients (solid bar).

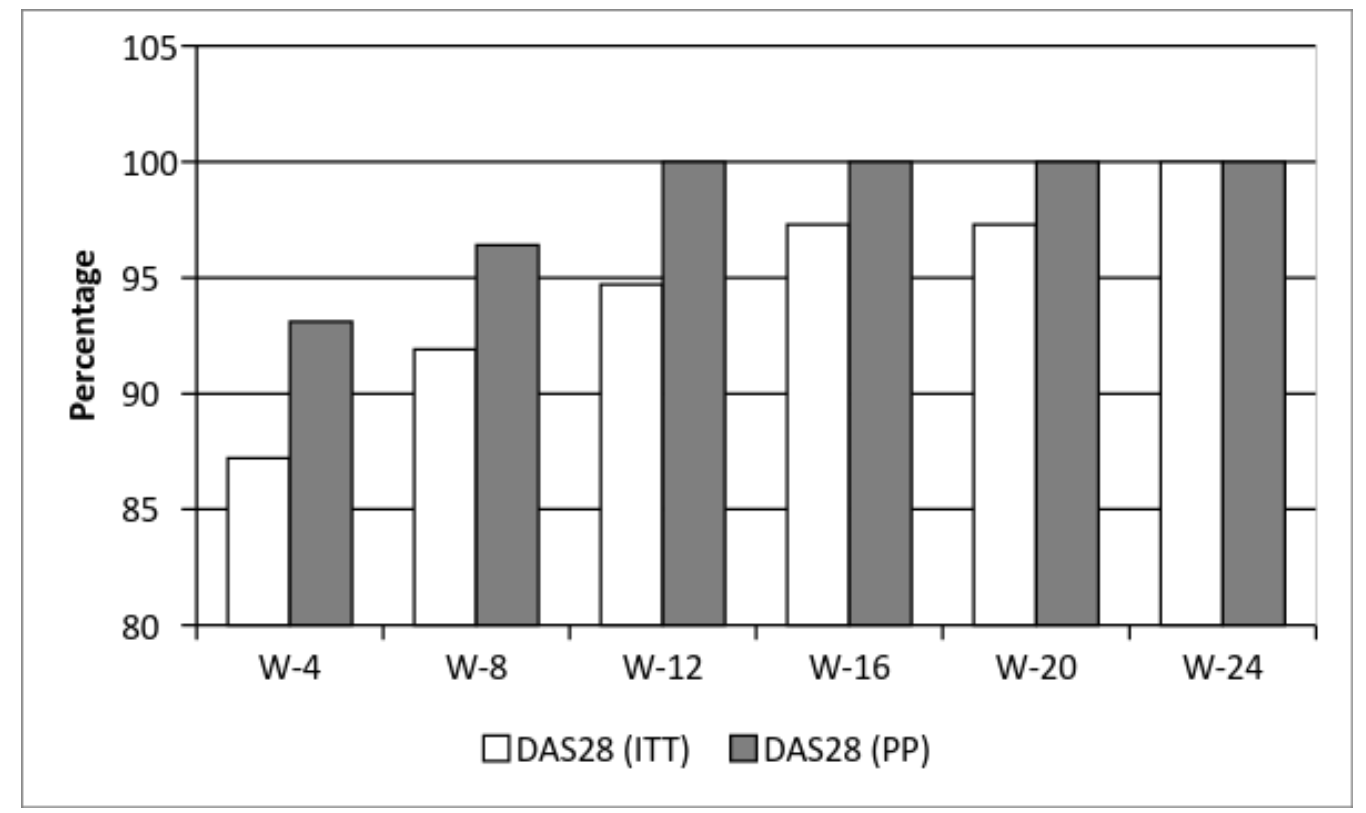

Figure 4. Percentage of patients achieving remission (DAS28 <2.6) in each visit.

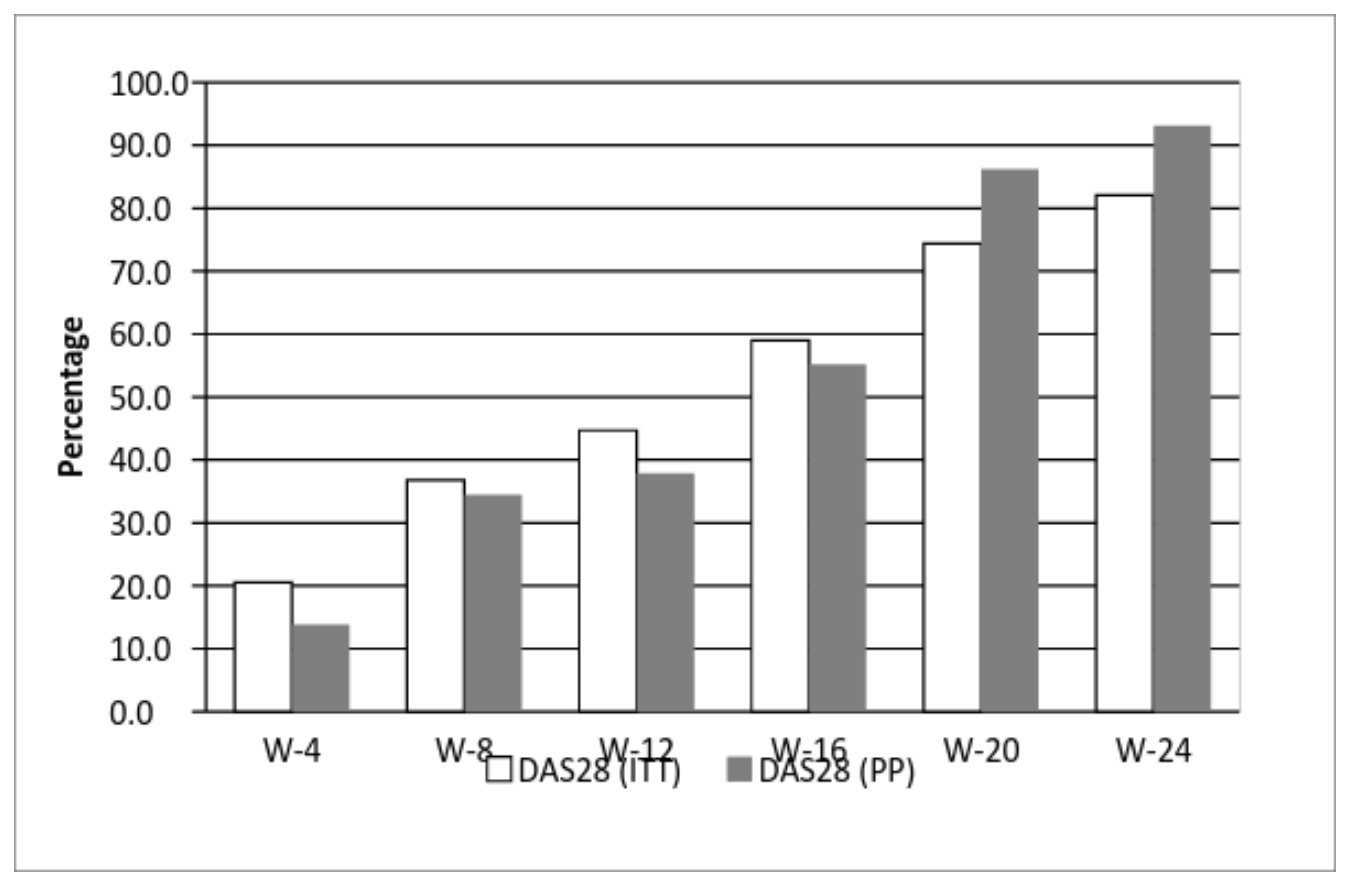


Figure 5. Percentage of patients with ACR20, ACR50, and ACR70 response at week 24 (ITT and PP)

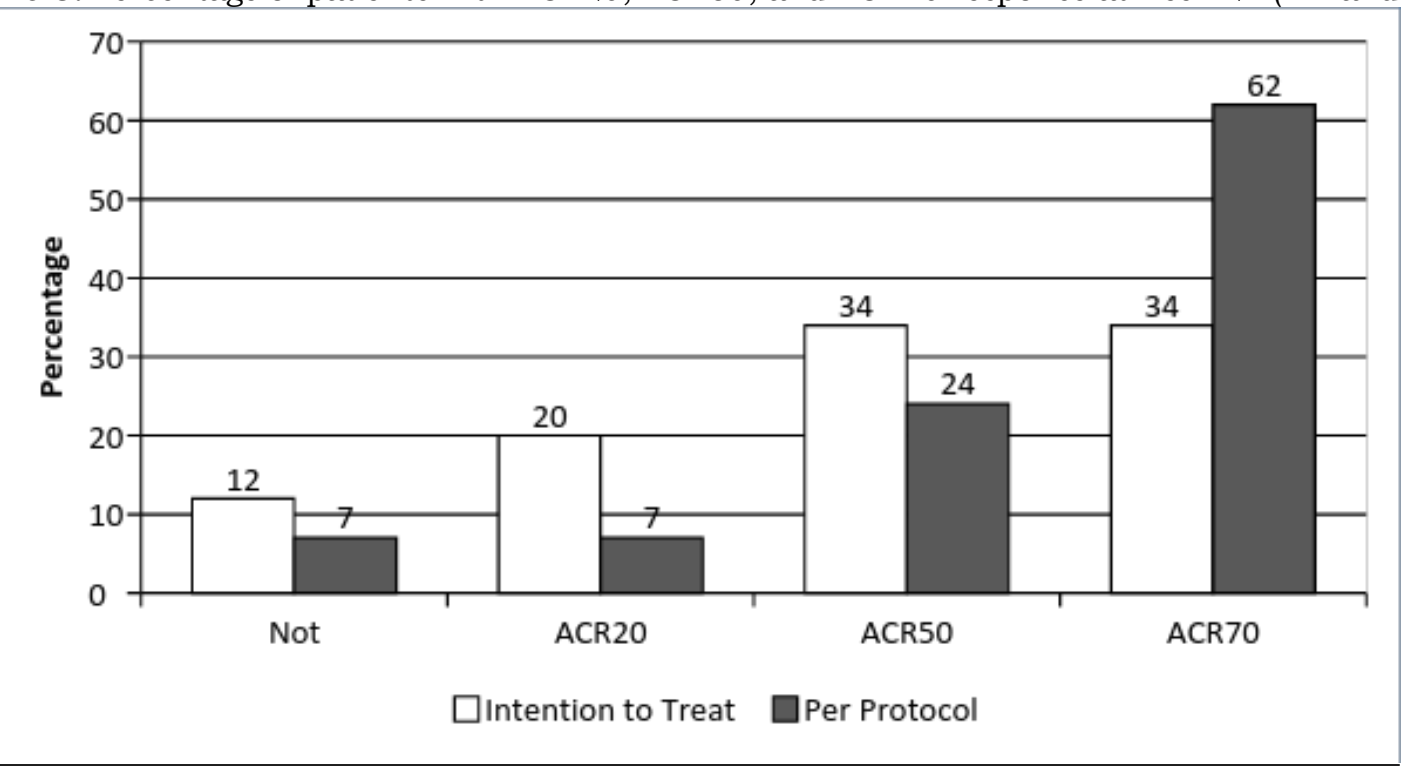

Figure 6. ESR and CRP at every visit during the treatment with tocilizumab and metothrexate.

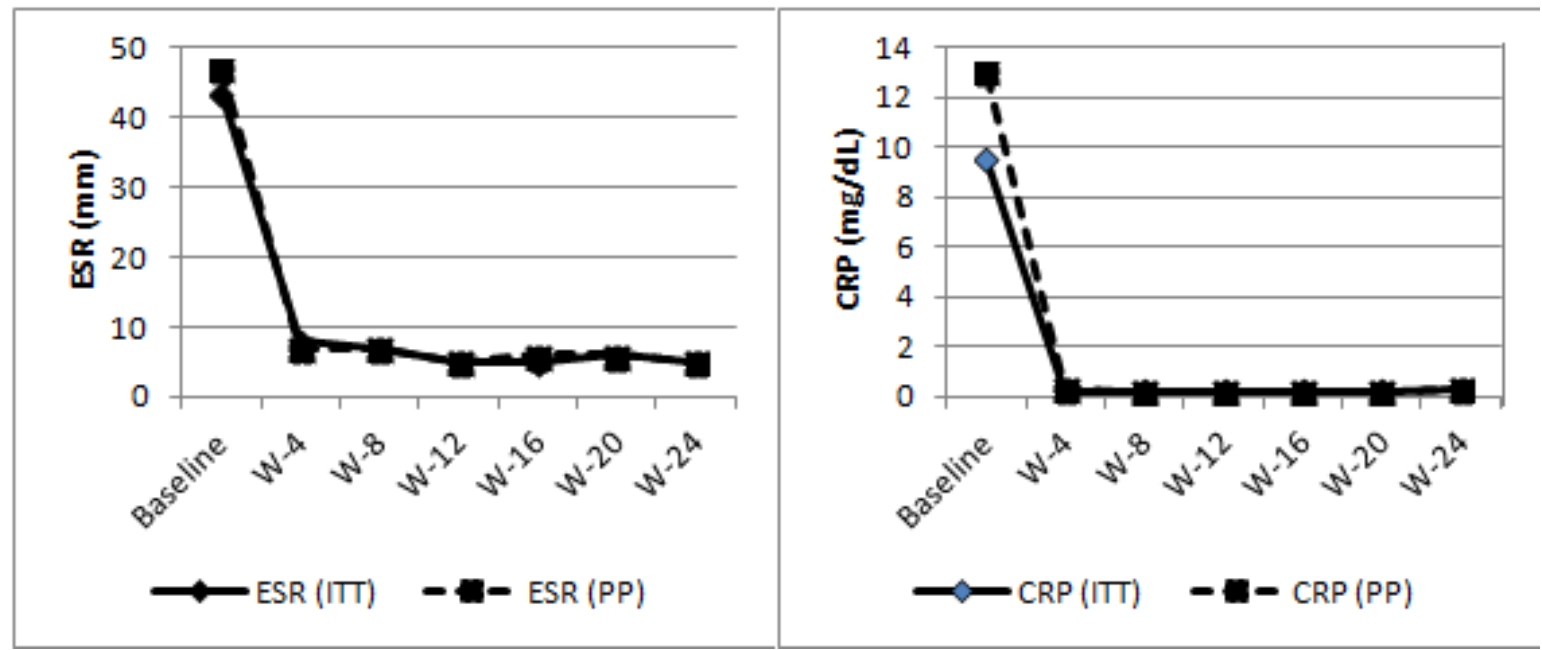

Table 3. Adverse events during administration of TCZ in combination with MTX.

\begin{tabular}{llll}
\hline No & Adverse Events & $\mathbf{N}$ & $\mathbf{\%}$ \\
\hline 1 & Increased hepatic enzyme & 12 & 30.7 \\
2 & Hypercholesterolemia & 9 & 23.1 \\
3 & Arthralgia & 8 & 20.5 \\
4 & Extremity Pain & 6 & 15.4 \\
5 & Ankle pain & 5 & 12.8 \\
6 & Stomatitis & 5 & 12.8 \\
7 & Cough & 3 & 7.7 \\
8 & Dyspepsia & 3 & 7.7 \\
9 & Headache & 3 & 7.7 \\
10 & Influenza & 3 & 7.7 \\
\hline
\end{tabular}


Figure 7. Number of patients with elevations in lipid.

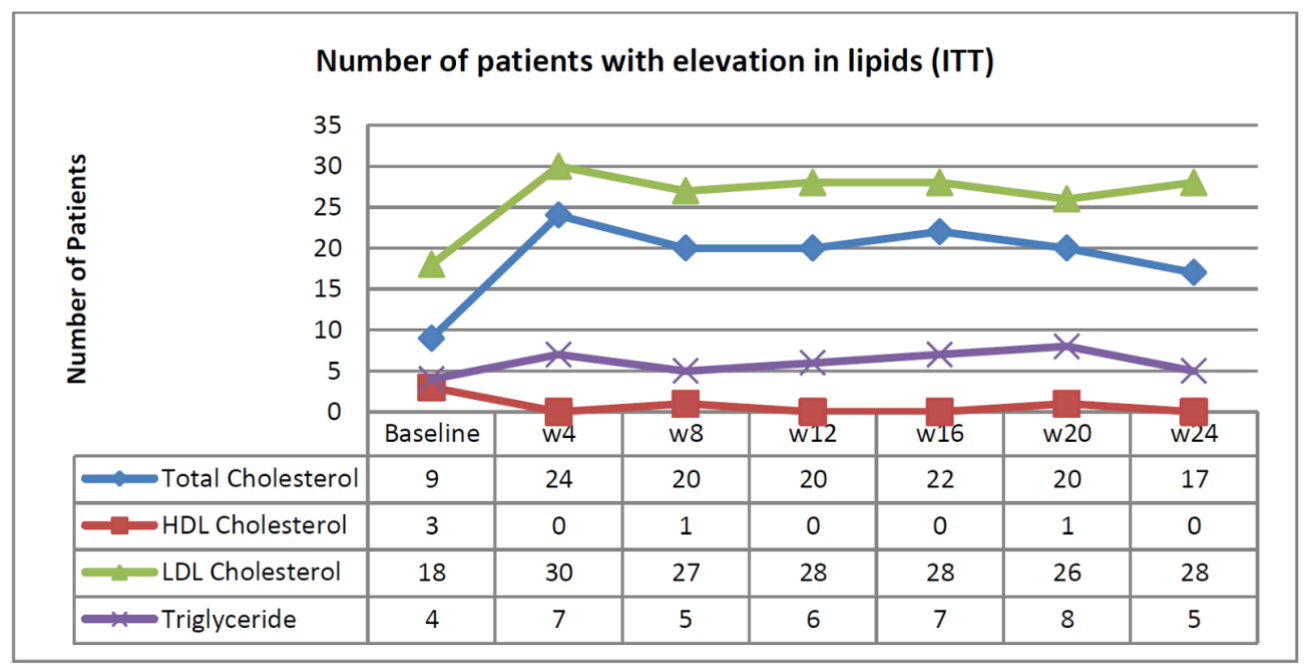

\section{DISCUSSION}

This was an interventional study with single arm to assess the efficacy of tocilizumab (TCZ) in combination with methotrexate (MTX) in Indonesian patients with active rheumatoid arthritis who have an inadequate response to non-biologic DMARDs. The limitations of this study included a non-comparative study design and small number of subjects. Fifty eight patients were screened but only 39 patients were enrolled to this study and included as ITT population. Four patients were withdrawn during the study due to SAE/AE (3 patients) and treatment failure (1 patient). There were 7 patients had protocol violations related with the entry criteria (eligibility criteria). Six patients out of those 7 ineligible patients had completed the study until week 24 due to delay in identifying the this protocol deviation related to the eligibility criteria while the other 1 patient was one of the four patients withdrawn during the study. Thus, only 29 patients were included in the per protocol (PP) population (refer to figure 2). However, the overall results of this study show that treatment with TCZ, alone or in combination with MTX, is associated with significant improvements of RA symptoms compared to non-biologic DMARDS that have been received by the subjects. In addition, results of this treatment showed a favorable safety profile.

Rheumatoid arthritis is a chronic autoimmune inflammatory disease with a tendency of progression. Control of this disease is often problematic due to progressive nature of the disease. Spontaneous remission is rare, although still possible. TCZ represents a novel approach to the treatment of RA and, therefore, may offer an alternative for patients who have had an inadequate clinical response to nonbiologic DMARDs. Overall the benefit/risk ratio for tocilizumab treatment in patients with moderate to severe RA is considered positive. These data provide the basis for clinical practice recommendations to Indonesian physicians on the management of patients treated with TCZ.

As based on efficacy result in this study, 100\% patients reached low disease activity (DAS28 $\leq 3.2$ ) and a clinically significant improvement (reduction at least 1.2 units at every visit) in DAS28 on last study visit (week 24) both for ITT or PP patient populations. Although this was a non-comparative study, this significant improvement is unlikely attributable to either carry over effect of MTX or spontaneous improvement. This statement is supported by the fact that all patients have been inadequately responsive to DMARDs after 8 weeks of stable dose. The rapid improvement after $\mathrm{TCZ}$ administration indicates that TCZ does have significant contribution to this improvement. 


\section{Clinical remission}

In addition, on last study visit, most of patients reached clinical remission (DAS28<2.6), 82.1\% in ITT population and $93.1 \%$ in PP population.

The study by Jones et al compared the effect of MTX with TCZ and placebo in 673 RA patients. It was reported that after 24 weeks, TCZ gave significantly better outcome in term of ACR2 0 and DAS28 < 2.6. A study with similar methodology has been conducted by Genovese et al. Proportion of patients achieving ACR20 response at week 24 was significantly greater in the TCZ plus DMARD group compared to control group, and combination of TCZ with other DMARD was shown to be better than DMARD alone. A double blind randomized control trial comparing TCZ alone or in combination with MTX or MTX alone in European RA patients with inadequate response to DMARDs has been reported by Maini et al. ${ }^{5}$ Statistically significant ACR50 and ACR70 responses were observed in patients receiving combination therapy with either $4 \mathrm{mg} / \mathrm{kg}$ or $8 \mathrm{mg} / \mathrm{kg}$ of TCZ plus MTX. SURPRISE study by the group of Kaneko et al reported that tocilizumab treatment added to methotrexate more rapidly suppressed inflammation than switching from methotrexate to tocilizumab. ${ }^{13}$ Regarding to other biologic non-TNF drugs, tocilizumab shows highest performance compared to rituximab, abatacept and tofacitinib in patients with RA. ${ }^{14}$

In our study, ACR20, ACR50 and ACR70 have been achieved in 20\%, 34\% and $34 \%$ by ITT patients, respectively, while on PP patients, the results were $7 \%$, $24 \%$, and $62 \%$. It means that the longer the administration of TCZ, the better the outcome is.

\section{ESR and CRP at every visit}

After one dose of TCZ, the ESR and CRP were rapidly normalized in this study, signifying the efficacy of TCZ in controlling inflammation of the patients. Again in these cases, the effects on ESR and CRP are unlikely to be due to MTX, since this non-biologic DMARD had previously been administered for at least eight weeks, yet the ESR and CRP remained elevated. Only after addition of TCZ those parameters normalized. This statement is supported by the study of Smolen et al, 12 which reported a rapid decrease of CRP level after administration of $8 \mathrm{mg} / \mathrm{kg}$ TCZ, while in a lower dose (4 $\mathrm{mg} / \mathrm{kg}$ ), the CRP re-increased after further observation, and it never decrease in placebo group.

\section{Safety profile}

Serious adverse events

Regarding that immunosuppressive agents including MTX and TCZ modify immune response of patients, the risk of infection in patients receiving these agents should be taken into account. Administration of MTX for at least 8 weeks followed by addition of TCZ for further 20 weeks has been associated with several incidences of serious infection. There were 3 out of 39 patients (7.69\%) had adverse events (AE) and serious adverse events (SAE) which caused discontinuation of TCZ treatment. SAE occurred in 2 patients $(<5 \%), 1$ patient experienced sepsis ec acquired community pneumonia and 1 patient experienced pneumonia tuberculosis. Other study by Jones et $\mathrm{al}^{11}$ reported an incidence of serious infection was comparable in group receiving TCZ $8 \mathrm{mg} / \mathrm{kg}$ or MTX (1.4\% vs $0.7 \%$ ).

\section{Other adverse events}

It can be seen in table 4 that increased liver enzyme represents the most frequent adverse event (30.7\%) followed by hypercholesterolemia (23.1\%). Increased total cholesterol, LDL-cholesterol has been observed, suggesting a precaution for increased risk for cardiovascular disease. Thus, lipid profile of patients, especially those with cardiovascular risk factors, should be taken into account before giving tocilizumab.

Twelve percent of patients needed dose modification due to elevated liver enzymes. Liver enzyme levels had reached normal value in all patients since week 20 . This incidence of increased liver enzyme is consistent with other TCZ phase III, randomized, double blind trials, OPTION study,15 AMBITION study, ${ }^{11}$ and TOWARD study. ${ }^{12}$ 
For overall monitoring of vital sign it was observed that there was no significant difference in vital sign parameter (blood pressure, body temperature, pulse rate) from time to time.

\section{Conclusion}

Tocilizumab in combination with methotrexate seems to be efficacious and likely to have good safety profile in RA patients of PICTURE INA Study in Indonesia. Patient screening and effective monitoring for efficacy and safety is essential in order to get optimal results.

\section{Acknowledgement}

This study is funded by PT. Roche Indonesia. The authors gratefully acknowledge the assistance of Dr. Nafrialdi in preparing the manuscript and Dr. Joedo Prihartono, MPH for providing statistical analysis.

\section{REFERENCES}

1. Silman AJ. Epidemiology and the rheumatic diseases. In: Maddison PJ, Isenberg DA, Woo P, Glass DN, eds. Oxford Textbook of Rheumatology: Oxford University Press:1993;499-513.

2. Brooks PM. Clinical management of rheumatoid arthritis. Lancet. 1993;341:286-290.

3. Edmonds JP, Scott DL, Furst DE, Brooks P, Paulus HE. Antirheumatic drugs: a proposed new classification. Arthritis Rheum 1993; 36:336-339.

4. Bathon JM, Martin RW, Fleischmann RM, Tesser JR, Schiff MH, Keystone EC, et al. A comparison of etanercept and methotrexate in patients with early rheumatoid arthritis. N Engl J Med. 2000; 343:1586-1592.

5. Maini RN, Taylor PC, Szechinski J, Pavelka K, Broll J, Balint G, et al. CHARISMA Study Group. Double-blind randomized controlled clinical trial of the interleukin-6 receptor antagonist, tocilizumab, in European patients with Rheumatoid arthritis who had an incomplete response to methotrexate. Arthritis Rheum 2006; 54:2817-2829.

6. Hirano T, Matsuda T, Turner M, Miyasaka N, Buchan G, Tang B, et al. Excessive production of IL-6/B cell stimulatory factor-2 in rheumatoid arthritis. Eur J Immunol 1988; 18:1797-801.
7. Keller TK, Wanagat J, Erschler WB. Molecular and cellular biology of interleukin-6 and its receptor. Frontiers Biosci 1996; 1:340-57.

8. Tamura T, Udagawa N, Takahashi N, Miyaura C, Tanaka S, Yamada Y, et al. Soluble interleukin-6 receptor triggers osteoclast formation by interleukin-6. Proc Natl Acad Sci USA 1993; 90:11924-11928.

9. The American Rheumatism Association 1987 revised criteria for the classificationof rheumatoid arthritis.Arthritis Rheum 1988;31:315-24

10. Van der Heijde DM, van't Hof MA, van Riel PL, Theunisse LA, Lubberts EW, van Leeuwen MA, et al. Judging disease activity in clinical practice in rheumatoidarthritis. First step in the development of a 'disease activity score'. Ann RheumDis 1990; 49:916-920

11. Jones G, Sebba A, Gu J, Lowenstein M B, Calvo A, Gomez-Reino $\mathrm{J} \mathrm{J}$ et al. Comparison of tocilizumab monotherapy versus methotrexate monotherapy in patients with moderate to severe rheumatoid arthritis: the AMBITION study. Ann Rheum Dis 2010;69:88-96. doi:10.1136/ard.2008.105197

12. Genovese MC, McKay JD, Nasonov EL, Mysler E F, da Silva N A, Alecock E, et al. Interleukin-6 Receptor Inhibition WithTocilizumab Reduces Disease Activity in Rheumatoid Arthritis With Inadequate Response to Disease-Modifying Antirheumatic Drugs. ARTHRITIS \& RHEUMATISM. 2008; 58(10):2968-80. DOI 10.1002/art.23940

13. Kaneko Y, Atsumi T, Tanaka Y et al. Comparison of adding tocilizumab to methotrexate with switching to tocilizumab in patients with rheumatoid arthritis with inadequate response to methotrexate: 52-week results from a prospective, randomised, controlled study (SURPRISE study). Ann Rheum Dis 2016;0:1-7. doi:10.1136/annrheumdis-2015-208426

14. Lee YH and Bae SC. Comparative efficacy and safety of tocilizumab, rituximab, abatacept and tofacitinib in patients with active rheumatoid arthritis that inadequately responds to tumor necrosis factor inhibitors: a Bayesian network meta-analysis of randomized controlled trials. Int J Rheumatic Dis.2015;1-9

15. Smolen J S, Beaulieu A, Rubbert-Roth A, RamosRemus C, Rovensky J, Alecock E, et al. forthe OPTION Investigators. Effect of interleukin-6 receptor inhibition with tocilizumabin patients with rheumatoid arthritis (OPTION study):a double-blind, placebo-controlled, randomised trial. Lancet 2008; 371: 987-97 